International Mathematical Forum, Vol. 9, 2014, no. 29, 1383 - 1388

HIKARI Ltd, www.m-hikari.com

http://dx.doi.org/10.12988/imf.2014.47143

\title{
Some Properties About R-Polynomials ${ }^{1}$
}

\author{
Yanni Zhou, Xigou Zhang and Zhaojin Zhou \\ College of Mathematics and Information Science \\ JXNU Nanchang, P.R. China, 330022
}

Copyright (c) 2014 Yanni Zhou, Xigou Zhang and Zhaojin Zhou. This is an open access article distributed under the Creative Commons Attribution License, which permits unrestricted use, distribution, and reproduction in any medium, provided the original work is properly cited.

\begin{abstract}
In this paper, we consider the special value about $\mathrm{R}$-polynomials in finite coxeter groups. Knowing $R_{x, w}=(q-1)^{l(w)-l(x)}$ where $x \leq w$, if $l(w)-l(x) \leq 2$, now we give the fact that there exist some $s, w$ such that $R_{x, w}=(q-1)^{l(w)-l(x)}$, where $x \leq w$, when $l(w)-l(x) \geq 3$. In particular, we discuss some special cases.
\end{abstract}

Keywords: the R-polynomial; coxeter groups; Hecke algebras;dihedral group

\section{Introduction}

$A$ Coxeter System is a pair $(W, \mathcal{S})$ consisting of a group $W$ and a set of generators $\mathcal{S}$, subject only to relations of the form $\left(s s^{\prime}\right)^{m\left(s, s^{\prime}\right)}=1$, where $m(s, s)=1, m\left(s, s^{\prime}\right)=m\left(s^{\prime}, s\right) \geq 2$ for $s \neq s^{\prime}$ in $\mathcal{S}$.

An arbitrary $w \in W$ can be written as a product of elements in $\mathcal{S}$, say $w=s_{1} \ldots s_{r}$ (where $\left.s_{i} \in \mathcal{S}\right)$. Define the length $l(w)$ of $w$ to be the smallest $r$ for which such an expression exists, and call the expression reduced.

Lemma 1. (see [1]) Let $s \in S, w \in W$ satisfy sw $<w$. Suppose $x<w$.

(a) If $s x<x$, then $s x<s w$.

(b) If $s x>x$, then $s x \leq w$ and $x \leq s w$.

Thus, in either case, $s x \leq w$.

\footnotetext{
${ }^{1}$ This work was supported in part by the NSF of P.R.China(NO: 11261021). The author was also supported in part by the Science Foundation of Education Department of JX Province(NO:GJJ10396).
} 
We begin with a very general construction of associative algebras over a commutative ring $A$ (with1). Such an algebra will have a free $A$-basis parameterized by the element of $W$, together with a multiplication law which reflects in a certain way the multiplication in $W$. The algebra will also depend on some parameters $a_{s}, b_{s} \in A(s \in \mathcal{S})$, subject only to the requirement that $a_{s}=b_{t}$ and $b_{s}=b_{t}$ whenever $s$ and $t$ are conjugate in $W$. The starting point for the construction is a free $A$ - module $\varepsilon$ on the set $W$, with basis elements denoted $T_{w}(w \in W)$ which satisfy the following.

$$
\begin{aligned}
& T_{s} T_{w}=T_{s w} \quad \text { if } s w>w, \\
& T_{s} T_{w}=a_{s} T_{w}+b_{s} T_{s w} \quad \text { if } s w<w .
\end{aligned}
$$

Now let $A$ be the ring $Z\left[q, q^{-1}\right]$ of Laurent polynomials over $Z$ in the indeterminate $q$. With the further convention that $a_{s}=q-1$ and $b_{s}=q$ for all $s \in \mathcal{S}$, we write $\mathcal{H}$ for the resulting generic algebra and call it the Hecke algebra of $W$.

Proposition 2. (see [1]) For all $w \in W$,

$$
\left(T_{w}^{-1}\right)^{-1}=\varepsilon_{w} q_{w} \sum_{x \leq w} \varepsilon_{x} R_{x, w}(q) T_{x},
$$

where $R_{x, w} \in Z[q]$ is a polynomial of degree $l(w)-l(x)$ in $q$, and where $R_{w, w}(q)=1, \varepsilon_{w}=(-1)^{l(w)}, q_{w}=q^{l(w)}$.

We can know the algorithm for computing $R_{x, w}$ implied by the proof of Proposition 2. The idea is to use induction on $l(w)$, starting with the fact that $R_{w, w}=1$ for all $w \in W$, while $R_{x, w}=0$ unless $x \leq w$. For the induction step, we need to compute $R_{x, w}$, assuming that all polynomials $R_{y, z}$ are known for $l(z)<l(w)$. Fix $s \in S$ for which $s w<w$. Then two configurations have to be dealt with, as in Lemma 1:

(A) $x<w, s x<x$ (forcing $s x<s w$ ). Here we found that $R_{x, w}=R_{s x, s w}$, which is already known since $s w<w$.

(B) $x<w, x<s x$ (forcing $s x \leq w$ and $x \leq s w$ ). Here we found that $R_{x, w}=(q-1) R_{x, s w}+q R_{s x, s w}$, both terms of which are already known. (Recall that the first term has degree $l(w)-l(x)$, while the second term has lower degree and might be 0 .)

It is sometimes useful to have alternate versions of (A) and (B), with $s$ occurring on the right rather than the left. For the right-handed version, we have the relations as follows:

(C) $x<w, x s<x, w s<w$ (forcing $x s<w s$ ). Then $R_{x, w}=R_{x s, w s}$.

(D) $x<w, x<x s$ (forcing $x s \leq w$ and $x \leq w s$ ). Then $R_{x, w}=(q-1) R_{x, w s}+$ $q R_{x s, w s}$.

The R-polynomials are built up, we consider the special case $l(w)-l(x)=1$. If $w=s_{1} \ldots s_{r}$ is a reduced expression, we can obtain $x$ by omitting a single $w=s_{i}, x=1$. As remarked at the beginning of the proof of Proposition 2, we get $R_{x, w}=q-1$. 
To carry this a step further, consider what happens when $l(w)-l(x)=2$. Fixing as before a reduced expression for $w$, we observe that (for reason of parity) $x$ can be obtained by omitting precisely two of the factors $s_{i}, s_{j}(i<j)$. Again we can apply (A) and (C) repeatedly to reduce to the case: $w=s_{i} \ldots s_{j}$, $x=s_{i+1} \ldots s_{j-1}$. Taking $s=s_{i}$, we have the configuration: $s w<w, x<s x$. Therefore (B) applies and we have $R_{x, w}=(q-1) R_{x, s w}+q R_{s x, s w}$. The first term is known from the preceding calculation: $R_{x, w}=q-1$. On the other hand, both $s x$ and $s w$ have the same length but are unequal, forcing the second term to be 0 . Conclusion: $R_{x, w}=(q-1)^{2}$.

The intrepid reader may wish to press on with these explicit calculations. However, they rapidly become less manageable, become of the more complicated possibilities for subexpressions when more than two factors are omitted. For example, when $W=A_{2}$, let $x=1, w=w_{0}$ (the longest element) in $W$, $R_{x, w}=(q-1)^{3}+q(q-1)$.

Enumerate a simple system $\Delta$ as $\alpha_{1}, \ldots, \alpha_{n}$, with corresponding simple reflections $s_{1}, \ldots, s_{n}$. Then $s_{1} \ldots s_{n}$ is called a Coxeter element of $W$. It depends on the choice of $\Delta$ as well as on the way $\Delta$ is numbered.

As promised above, we discuss briefly some of $R_{x, w}$ which satisfy $R_{x, w}=$ $(q-1)^{l(w)-l(x)}$. Consider that $W=A_{3}$ with corresponding simple reflections $s_{1}$, $s_{2}, s_{3}$, we can compute all the $R_{x, w}$, it will be clear to find that $R_{x, w}=(q-1)^{3}$, where $w$ is any Coxeter element of $W$. In this paper, we wish to obtain some results about the $\mathrm{R}$-polynomials in finite Coxeter groups.

\section{Main results and their proofs}

Proposition 3. Let $I \subseteq S$, write $S=\left\{s_{1}, s_{2}, \ldots, s_{n}\right\}, I=\left\{s_{i_{1}}, s_{i_{2}}, \ldots, s_{i_{r}}\right\}$ and let $J$ consist of all Coxeter elements of $W_{I}$. We have $R_{1, w}=(q-1)^{l(w)}$ for any $w \in J$.

Proof. Proceed by induction on $l(w)$, assuming that $w=s_{i_{1}} s_{i_{2}} \ldots s_{i_{r}}$, this is clear when $l(w)=0,1$ or 2 . Let $3 \leq l(w)<n$ (or equivalently, $3 \leq r<n$ ) such that $R_{1, w}=(q-1)^{l}(w)$. If $l(w)=\mathrm{n}$ (or equivalently, $\left.r=n\right)$. Then

$$
R_{1, w}=R_{1, s_{i_{1}} s_{i_{2}} \ldots s_{i_{r}}}=(q-1) R_{1, s_{i_{2}} \ldots s_{i_{r}}}+q R_{s_{i_{1}}, s_{i_{2}} \ldots s_{i_{r}}} .
$$

Since $l\left(s_{i_{2}} \ldots s_{i_{r}}\right)=n-1<n$, by induction

$$
R_{1, s_{i_{2}} \ldots s_{i_{r}}}=(q-1)^{n-1} \text {. }
$$

Since $i_{1} \neq i_{2} \neq \ldots \neq i_{n}$, it implies that $s_{i_{1}}$ is not the subsequence of $s_{i_{2}} \ldots s_{i_{r}}$, we can obtain

Finally

$$
R_{s_{i_{1}}, s_{i_{2}} \ldots s_{i_{r}}}=0
$$

$$
R_{1, w}=(q-1)(q-1)^{n-1}=(q-1)^{l(w)},
$$

as requird. 
Corollary 4. Let $I \subseteq S$, write $S=\left\{s_{1}, s_{2}, \ldots, s_{n}\right\}, I=\left\{s_{i_{1}}, s_{i_{2}}, \ldots, s_{i_{r}}\right\}$ and let $J$ consist of all Coxeter elements of $W_{I}$. Finding $w_{1} \in W$ and $w \in J$ such that $l\left(w_{1} w\right)=l\left(w_{1}\right)+l(w)$ (resp. $\left.l\left(w w_{1}\right)=l(w)+l\left(w_{1}\right)\right)$, we have $R_{w_{1}, w_{1} w}=(q-1)^{l(w)}$ (resp. $\left.R_{w_{1}, w w_{1}}=(q-1)^{l(w)}\right)$.

Proof. According to Proposition 3 and (A), it is clear that $R_{w_{1}, w_{1} w}=R_{1, w}=$ $(q-1)^{l(w)}$.

Proposition 5. Let $I \subseteq S$, write $S=\left\{s_{1}, s_{2}, \ldots, s_{n}\right\}, I=\left\{s_{i_{1}}, s_{i_{2}}, \ldots, s_{i_{r}}\right\}$, where $r \geq 3$, and let $J$ consist of all Coxeter elements of $W_{I} . w=s_{i_{2}} \ldots s_{i_{1}} \ldots s_{i_{r}}$ for $w \in J$, then $R_{s_{i_{1}}, w}=(q-1)^{r-1}$.

Proof. We argue by induction on $l(w)$, starting with the fact that $l(w)=3$.

Hence

$$
R_{s_{i_{1}}, s_{i_{2}} s_{i_{1}} s_{i_{3}}}=(q-1) R_{s_{i_{1}}, s_{i_{1}} s_{i_{3}}}+q R_{s_{i_{2}} s_{i_{1}}, s_{i_{1}} s_{i_{3}}}=(q-1)^{2} .
$$

Consider the case $l(w)>3$, we can find $s=s_{i_{2}}$ such that $s w<w, w$ has two possibilities:

(a) If $w=s_{i_{2}} s_{i_{1}} \ldots s_{i_{r}}$, we have

$$
R_{s_{i_{1}}, w}=(q-1) R_{s_{i_{1}}, s_{i_{2}} w}+q R_{s_{i_{2}} s_{i_{1}}, s_{i_{2}} w}=(q-1)^{r-1} .
$$

(b) If $w=s_{i_{2}} s_{i_{3}} \ldots s_{i_{1}} \ldots s_{i_{r}}$, we have

$$
R_{s_{i_{1}}, w}=R_{s_{i_{1}}, s_{i_{2}} s_{i_{3}} \ldots s_{i_{1}} \ldots s_{i_{r}}}=(q-1) R_{s_{i_{1}}, s_{i_{3}} \ldots s_{i_{1}} \ldots s_{i_{r}}}+q R_{s_{i_{2}} s_{i_{1}}, s_{i_{3}} \ldots s_{i_{1}} \ldots s_{i_{r}}}
$$

By the induction hypothesis, we have

$$
R_{s_{i_{1}}, s_{i_{3}} \ldots s_{i_{1}} \ldots s_{i_{r}}}=(q-1)^{r-2} .
$$

Since $s_{i_{2}} s_{i_{1}}$ is not the subsequence of $s_{i_{3}} \ldots s_{i_{1}} \ldots s_{i_{r}}$, thus

$$
R_{s_{i_{2}} s_{i_{1}}, s_{i_{3}} \ldots s_{i_{1}} \ldots s_{i_{r}}}=0 \text {. }
$$

Combining these, we have

$$
R_{s_{i_{1}}, w}=(q-1)^{r-1},
$$

as required.

Corollary 6. Let $I \subseteq S$, write $S=\left\{s_{1}, s_{2}, \ldots, s_{n}\right\}, I=\left\{s_{i_{1}}, s_{i_{2}}, \ldots, s_{i_{r}}\right\}$ and let $J$ consist of all Coxeter elements of $W_{I}$.

(a) Assume that there exists $s=s_{i_{j}}$ such that $s w>w$ (resp. ws $\left.>w\right)$, where $1 \leq j \leq r$, then $R_{1, s w} \neq(q-1)^{l(w)}$ (resp. $\left.R_{1, w s} \neq(q-1)^{l(w)}\right)$ for $w \in J$.

(b) Let $w=s_{i_{1}} \ldots s_{i_{r}}$ for $w \in J$, assume that there exists $s=s_{i_{j}}$ such that $s_{i_{1}} \ldots s \ldots s_{i_{r}}>s_{i_{1}} \ldots s_{i_{r}}$, where $1 \leq j \leq r$, then $R_{1, s_{i_{1}} \ldots s \ldots s_{i_{r}}} \neq(q-1)^{r+1}$.

Proof. Assume that $w=s_{i_{1}} s_{i_{2}} \ldots s_{i_{r}}$ which implies $s \neq s_{i_{1}}$, we have

$$
R_{1, s w}=(q-1) R_{1, w}+q R_{s, w} .
$$

By proposition 3, we can obtain $R_{1, w}=(q-1)^{l(w)}$. By proposition 5, we get $R_{s, w}=(q-1)^{l(w)-1}$. Combine these, we compute

$$
R_{1, s w}=(q-1) R_{1, w}+q R_{s, w}=(q-1)^{l(w)+1}+q(q-1)^{l(w)-1},
$$


this proves (a).

Suppose that $s$ occurs in the $m$ th position of $R_{1, s_{i_{1}} \ldots s \ldots s s_{i}}$, where $m$ satisfies $1<m \leq\left[\frac{r+1}{2}\right]+1$, if $r$ is even, $1<m \leq \frac{r+1}{2}$, if $r$ is odd. Proceed by induction on $m$, if $m=2$, then

$$
R_{1, s_{i_{1}} s s_{i_{2}} \ldots s_{i_{r}}}=(q-1) R_{1, s s_{i_{2}} \ldots s_{i_{r}}}+q R_{s_{i_{1}}, s s_{i_{2}} \ldots s_{i_{r}}}
$$

Since $s_{i_{1}} \ldots s \ldots s_{i_{r}}>s_{i_{1}} \ldots s_{i_{r}}$, it is clear that $s \neq s_{i_{1}}$, which implies that $s_{i_{1}}$ is not the subsequence of $s s_{i_{2}} \ldots s_{i_{r}}$, hence, $R_{s_{i_{1}}, s s_{i_{2}} \ldots s_{i_{r}}}=0$, however, by the case (a), we get $R_{1, s s_{i_{2}} \ldots s_{i_{r}}} \neq(q-1)^{r}$, thus $R_{1, s_{i_{1}} s s_{i_{2}} \ldots s_{i_{r}}} \neq(q-1)^{r+1}$.

Assume that $m=k$, where $1<k<\left[\frac{r+1}{2}\right]+1$, if $r$ is even, $1<k<\frac{r+1}{2}$, if $r$ is odd. The case (b) holds. Now $m=k+1$, so

$$
R_{1, s_{i_{1}} s_{i_{2}} \ldots s_{i_{k}} s s_{i_{k+1}} \ldots s_{i_{r}}}=(q-1) R_{1, s_{i_{2}} \ldots s_{i_{k}} s s_{i_{k+1}} \ldots s_{i_{r}}}+q R_{s_{i_{1}}, s_{i_{2}} \ldots s_{i_{k}} s s_{i_{k+1}} \ldots s_{i_{r}}}
$$

two cases are possible:

(i) Suppose that $s=s_{i_{1}}$, by Proposition 3 and by Proposition 5 , so we have $R_{1, s_{i_{2}} \ldots s_{i_{k}} s s_{i_{k+1}} \ldots s_{i_{r}}}=(q-1)^{r}$, while $R_{s_{i_{1}}, s_{i_{2}} \ldots s_{i_{k}} s s_{i_{k+1}} \ldots s_{i_{r}}}=(q-1)^{r-1}$. Hence

$$
R_{1, s_{i_{1}} s_{i_{2}} \ldots s_{i_{k}} s s_{i_{k+1}} \ldots s_{i_{r}}}=(q-1)^{r+1}+q(q-1)^{r-1},
$$

this proves (b).

(ii) Suppose that $s \neq s_{i_{1}}$, note that $s$ is not the subsequence of $s_{i_{2}} \ldots s_{i_{k}} s s_{i_{k+1}} \ldots s_{i_{r}}$, so we have $R_{s_{i_{1}}, s_{i_{2}} \ldots s_{i_{k}} s s_{i_{k+1}} \ldots s_{i_{r}}}=0$. On the other hand, by the induction hypothesis, we have $R_{1, s_{i_{2}} \ldots s_{i_{k-1}}} s s_{i_{k}} \ldots s_{i_{r}} \neq(q-1)^{r+1}$, it can deduce $R_{1, s_{i_{2}} \ldots s_{i_{k}} s s_{i_{k+1}} \ldots s_{i_{r}}} \neq$ $(q-1)^{r}$, this is to say, $R_{1, s_{i_{1}} \ldots s \ldots s_{i_{r}}} \neq(q-1)^{r+1}$, as required.

Proposition 7. Let $W$ be a dihedral group $D_{m}$, where $m<\infty$, if $l(w)-l(x) \geq$ 3 , then $R_{x, w} \neq(q-1)^{l(w)-l(x)}$.

Proof. In fact, we only consider two possibilities:

The case (a) that $x=1$, and $w=$ sts $\ldots$ (resp. $w=t s t \ldots)$ satisfy $l(w) \geq 3$, we have

$$
R_{1, w}=(q-1) R_{1, t s \ldots}+q R_{s, t s \ldots}
$$

It is clear that $R_{s, t s \ldots} \neq 0$, forcing $q$ is a factor of some term in $R_{1, w}$, hence $R_{1, w} \neq(q-1)^{l(w)}$.

The case (b) that $x=s t s t \ldots s$ and $w=t s t \ldots t$ satisfy $l(w)-l(x) \geq 3$, we have

$$
R_{x, w}=(q-1) R_{x, t w}+q R_{t x, t w} .
$$

Since $l(w)-l(x) \geq 3$, we can get $l(t w)-l(t x) \geq 1$ and $t x \leq t w$, so $R_{t x, t w} \neq 0$, forcing $q$ is a factor of some term in $R_{x, w}$, hence $R_{x, w} \neq(q-1)^{l(w)-(x)}$.

In each case, $R_{x, w} \neq(q-1)^{l(w)-(x)}$, as required. 


\section{REFERENCES}

[1] James E Humphreys. The reflection groups and Coxeter groups [M]. Cambridge:Cambridge University Press, 1990.

[2] Meinolf Geck, G. Pfeiffer. Characters of finite Coxeter groups and Iwahori-Hecke, Oxford:Clarendon Press, 2000.

[3] D. Kazhdan and G. Lusztig, Representations of Coxeter groups and Hecke algebras, Invent. Math. 53 (1979), 165-184.

[4] M. Dyer, Hecke algebras and reflections in Coxeter groups. Ph. D. thesis, University of Sydney, 1987.

[5] H. Wenzl, Hecke algebras of type $A_{n}$ and subfactors, Invent. Math. 92 (1988) 349.

[6] V.V.Deodhar, On Bruhat ordering and weight-lattice ordering for a Weyl group, Indag. Math. 40 (1978), 423-435.

Received: July 7, 2014 